\title{
9
}

\section{UN MODELO PREDICTIVO DEL RENDIMIENTO ACADÉMICO A PARTIR DE LAS CALIFICACIONES DE BACHILLERATO Y PAU}

\section{A PREDICTIVE MODEL OF ACADEMIC PERFORMANCE BASED ON HIGH SCHOOL GRADE POINT AVERAGE AND UNIVERSITY ACCESS TEST RESULTS}

\author{
Roberto Dorta Guerra \\ rodorta@ull.edu.es \\ Isabel Marrero \\ imarrero@ull.es \\ Beatriz Abdul-Jalbar \\ babdul@ull.edu.es \\ Rodrigo Trujillo González \\ rotrujil@ull.edu.es \\ Néstor Torres Darias \\ ntorres@ull.edu.es
}

Universidad de La Laguna, España

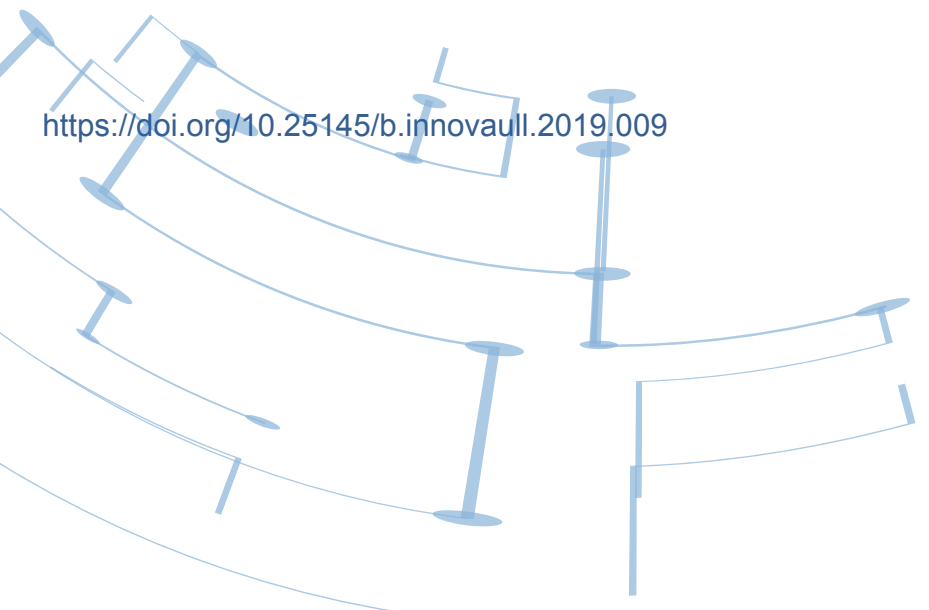




\section{RESUMEN}

Numerosas investigaciones educativas muestran que el rendimiento académico en el primer año de universidad incide en el éxito con el que se cursan los años subsiguientes, lo que justifica el interés de analizar el rendimiento, durante el primer curso, del alumnado de nuevo ingreso e identificar los factores que influyen en él. En el presente trabajo se ha definido un nuevo indicador de este rendimiento y se han determinado, para cada uno de los grados en Ciencias de la ULL, aquellos indicadores de rendimiento previo con los que se encuentra más correlacionado el indicador introducido. Se han obtenido así sendos modelos de regresión lineal multivariante que permiten predecir el rendimiento de un estudiante de nuevo ingreso en el primer cuatrimestre del primer curso de cada grado en función de su rendimiento en Bachillerato y PAU. En todos los grados de Ciencias, la variable predictora dominante ha resultado ser la nota media de Bachillerato. La bondad de ajuste de los modelos que utilizan el nuevo indicador supera ampliamente la de otros modelos preexistentes en la literatura.

El método es extensible a cualquier grado y universidad. Su aplicación sistemática permitiría definir y detectar perfiles de riesgo académico con el propósito de contribuir, por una parte, a que cada estudiante adopte una actitud proactiva hacia la subsanación de sus posibles deficiencias formativas y, por otra, a que el gestor universitario optimice los recursos humanos y materiales necesarios para mejorar el aprovechamiento académico de los estudiantes en situación de riesgo.

PALABRAS CLAVE: Transición a la universidad, éxito académico, grados en Ciencias, regresión lineal multivariante.

\section{ABSTRACT}

Several educational investigations have shown that the academic performance in the first year of university affects the success in subsequent years, which justifies the interest of analyzing the performance, during the first year, of new students in order to identify the factors that influence it. In the present work, a new indicator of this performance has been defined and those indicators of previous performance which are best correlated with the indicator introduced have been determined for each one of the degrees in Science of the ULL. We have thus obtained multivariate linear regression models that allow us to predict the performance of new students in the first semester of the first year of each degree, based on their performance in High School and the University Access Test. In all of Science degrees, the dominant predictor variable has turned out to be the High School grade point average. The goodness of fit of the models that use the new indicator far exceeds that of other pre-existing models in the literature.

Our method is extensible to any degree and university. Its systematic application would allow defining and detecting academic risk profiles so that, on the one hand, affected students may be encouraged to adopt a proactive attitude towards the correction of their training deficiencies and, on the other hand, university managers can optimize the human and material resources necessary to improve the academic performance of those students at risk.

KEYWORDS: Transition to university, academic success, Science degrees, multivariate linear regression. 


\section{INTRODUCCIÓN Y OBJETIVOS}

Tal como se acaba de señalar, entre las investigaciones educativas está consolidada la idea de que el rendimiento previo es un buen predictor del rendimiento futuro. En este trabajo presentamos un modelo predictivo del rendimiento del alumnado en función de su rendimiento previo. Para una primera aproximación al modelo, véase Trujillo et al. (2018).

El punto de partida es, necesariamente, la elección de un indicador de rendimiento adecuado. Tras estudiar exhaustivamente diversos indicadores propuestos en la literatura (cf. Bivin y Rooney, 1999; Mathiasen, 1984; Pike, 1991; Pike y Saupe, 2002; Zeegers, 2004; Zusho, Pintrich y Coppola, 2003), hemos optado por definir un nuevo índice cuya introducción se justifica por la creencia, comúnmente aceptada, de que un alumno tiene un rendimiento bajo cuando no aprueba ninguna asignatura o cuando sólo aprueba una. Definimos como nivel de rendimiento de un alumno la suma de las calificaciones de las asignaturas aprobadas, esto es, sólo de aquellas en las que su nota haya sido mayor o igual que 5. Técnicamente, se trata una variable de tipo cuantitativo que toma valores en el intervalo $[0,50]$, ya que todo el alumnado debe estar matriculado en las 5 asignaturas que se ofertan en el primer cuatrimestre de primer curso y la nota máxima de cada asignatura es un 10. Para un alumno con rendimiento bajo el valor máximo de esta variable es de 10 puntos ya que o no aprueba ninguna asignatura (en cuyo caso obtendría una calificación de 0), o sólo aprueba una (en la que, como máximo, podría obtener una puntuación de 10).

Una vez definido el indicador de rendimiento futuro, se pretende determinar cuáles son los indicadores de rendimiento previo con los que está más correlacionado y construir un modelo de regresión lineal multivariante que precise la relación existente. De esta manera, se obtiene una herramienta que permitiría predecir el rendimiento futuro de cada estudiante, haciéndole tomar conciencia de sus fortalezas y debilidades y creando las oportunas alertas para actuar en aquellos casos donde se detecten valores anormalmente bajos del indicador.

Como apéndice a este trabajo se presentan y discuten los perfiles y los resultados académicos del alumnado de nuevo ingreso en la Facultad de Ciencias (grados en Biología, Ciencias Ambientales, Física, Matemáticas y Química) de la ULL, para los cursos 2015/16 y 2016/17, en función de la nota de admisión. Tales resultados han motivado el desarrollo de los indicadores propuestos y serán comparados oportunamente con los que al término del curso 2017/18 obtenga la primera cohorte procedente del Bachillerato LOMCE y la EBAU. Las conclusiones de la comparativa servirán para adaptar a las sucesivas cohortes LOMCE los indicadores resultantes y diseñar planes de mejora del rendimiento académico en función de éstos. 


\section{MATERIAL Y MÉTODOS}

Participantes. Para cada uno de los grados de la Facultad de Ciencias la muestra de estudiantes incluye alumnos de nuevo ingreso en los cursos académicos 2015/16 y 2016/17. En concreto, las muestras comprenden a 79, 85, 81, 113 y 57 estudiantes del primer curso de los grados en Matemáticas, Química, Física, Biología y Ciencias Ambientales, respectivamente. Estas cifras son inferiores al número total de matriculados en cada grado, debido a que se eliminaron tanto los casos cuyos registros de la PAU estaban incompletos (pues las variables independientes que van a ser utilizadas guardan relación con dichos registros), como el alumnado con cero asignaturas presentadas (se considera que estos estudiantes o bien han cambiado de carrera, o que los estudios que comenzaron no cubren sus expectativas y han decidido abandonarlos).

Variable criterio en cada grado:

- Nivel de rendimiento del alumno $=$ Suma de las calificaciones del alumno para aquellas asignaturas aprobadas (nota de la asignatura $\geq 5$ ).

Variables explicativas en cada grado:

- Nota media del expediente en el Bachillerato (Media_expte).

- Nota de la materia de comentario de texto PAU (Notā text).

- Nota de la materia de lengua extranjera PAU (Nota_idioma).

- Nota de la materia común PAU (Nota_com).

- Nota de la materia de modalidad PAŪ (Nota_mod).

- Nota de la fase específica (Nota_fase_específica) = Nota de admisión al grado en que está matriculadō - Nota de acceso PAU.

Análisis. Para cada uno de los grados se lleva a cabo un modelo de regresión lineal múltiple con el objetivo de estudiar si existe relación entre la variable dependiente (nivel de rendimiento de un estudiante en el primer cuatrimestre de su primer año de carrera) y las variables independientes (nota de Bachillerato y calificaciones obtenidas en la PAU: nota del comentario de texto, nota de la lengua extranjera, nota de la materia común, nota de la materia de modalidad y nota de la fase específica). Una vez demostrado que, al menos, hay un conjunto de variables independientes relacionadas con el rendimiento futuro de un estudiante, se podrá utilizar el modelo obtenido para predecir ese rendimiento en función de dichas variables independientes. Para cada modelo se han verificado las hipótesis de linealidad, normalidad, homocedasticidad, independencia y ausencia de multicolinealidad. Además, la bondad de ajuste se evalúa a partir del coeficiente de determinación ajustado $R_{\mathrm{aj} j^{\prime}}^{2}$ el 
coeficiente de correlación múltiple $R$ y el estudio de la significación global del modelo. Asimismo, se analiza la significación individual de cada variable y se propone un método de selección de variables hacia adelante para determinar el modelo que mejor explica el rendimiento futuro de un alumno.

En todo el estudio se considera estadísticamente significativo un $p$ valor $<0,05$. Todos los análisis estadísticos fueron desarrollados haciendo uso del software estadístico IBM® SPSS, versión 21.

\section{RESULTADOS}

En las Tablas 1-5 se puede observar que, para todos los grados, la variable que se encuentra más correlacionada con el rendimiento futuro de un alumno es la nota media obtenida en el Bachillerato. Por lo tanto, se puede afirmar que esta variable es la que más contribuye a explicar el rendimiento futuro de los estudiantes en todos los grados.

\begin{tabular}{|c|c|c|c|c|c|c|}
\hline $\begin{array}{c}\text { Grado en } \\
\text { Matemáticas }\end{array}$ & $\begin{array}{l}\text { Rendi_- } \\
\text { alumno }\end{array}$ & $\begin{array}{c}\text { Media } \\
\text { expte }\end{array}$ & $\begin{array}{c}\text { Nota } \\
\text { text }\end{array}$ & $\begin{array}{l}\text { Nota_- } \\
\text { idioma }\end{array}$ & $\begin{array}{c}\text { Nota } \\
\text { com }^{-}\end{array}$ & $\begin{array}{l}\text { Nota } \\
\text { mod }\end{array}$ \\
\hline Media_expte & $0,798^{* * *}$ & & & & & \\
\hline Nota_text & $0,521^{* * *}$ & $0,569^{* * *}$ & & & & \\
\hline Nota_idioma & $0,437^{* * *}$ & $0,564^{* * *}$ & $0,563^{* * *}$ & & & \\
\hline Nota_com & $0,497^{* * *}$ & $0,535^{* * *}$ & $0,481^{* * *}$ & $0,368^{* * *}$ & & \\
\hline Nota_mod & $0,652^{* * *}$ & $0,541^{* * *}$ & $0,345^{* * *}$ & $0,289 * * *$ & $0,423^{* * *}$ & \\
\hline $\begin{array}{l}\text { Nota_fase_espe- } \\
\text { cífica }\end{array}$ & $0,676^{* * *}$ & $0,681^{* * *}$ & $0,428^{* * *}$ & $0,243^{* *}$ & $0,434^{* * *}$ & $0,452^{* * *}$ \\
\hline
\end{tabular}

${ }^{*} p<0,05 ;{ }^{* *} p<0,01 ;{ }^{* * *} p<0,001 ;$ ns: correlación no significativa.

TABLA 2: CoRrelaCiONES DE ORDEN CERO ENTRE El RENDIMIENTO EN EL PRIMER CUATRIMESTRE DE PRIMERO DEL Grado en Química y el RENDIMIENTO PREVIO (NOtAS DE Bachillerato y PAU)

\begin{tabular}{|l|c|c|c|c|c|c|}
\hline Grado en Química & $\begin{array}{c}\text { Rendi_ } \\
\text { alumno }\end{array}$ & $\begin{array}{c}\text { Media_- } \\
\text { expte }\end{array}$ & $\begin{array}{c}\text { Nota_- } \\
\text { text }\end{array}$ & $\begin{array}{c}\text { Nota__ } \\
\text { idioma }\end{array}$ & $\begin{array}{c}\text { Nota_- } \\
\text { com }^{-}\end{array}$ & $\begin{array}{c}\text { Nota } \\
\text { mod }\end{array}$ \\
\hline Media_expte & $0,718^{* * *}$ & & & & & \\
\hline Nota_text & $0,291^{* *}$ & $0,474^{* * *}$ & & & & \\
\hline Nota_idioma & $0,250^{*}$ & $0,352^{* * *}$ & $0,325^{* *}$ & & & \\
\hline Nota_com & $0,397^{* * *}$ & $0,427^{* * *}$ & $0,430^{* * *}$ & $0,104 \mathrm{~ns}$ & & \\
\hline Nota_mod & $0,454^{* * *}$ & $0,355^{* * *}$ & $0,291^{* *}$ & $0,211^{*}$ & $0,182^{*}$ & \\
\hline Nota_fase_específica & $0,512^{* * *}$ & $0,321^{* *}$ & $0,088 \mathrm{~ns}$ & $0,082 \mathrm{~ns}$ & $0,297^{* *}$ & $\begin{array}{c}0,064 \\
\mathrm{~ns}\end{array}$ \\
\hline
\end{tabular}

${ }^{*} \mathrm{p}<0,05 ;{ }^{* *} \mathrm{p}<0,01 ;{ }^{* * *} \mathrm{p}<0,001$; ns: correlación no significativa. 


\begin{tabular}{|c|c|c|c|c|c|c|}
\hline Grado en Física & $\begin{array}{l}\text { Rendi_- } \\
\text { alumno }\end{array}$ & $\begin{array}{l}\text { Media } \\
\text { expte }\end{array}$ & Next $_{\text {te }}^{\text {Not }}$ & $\begin{array}{l}\text { Nota } \\
\text { idioma }\end{array}$ & $\begin{array}{l}\text { Nota_} \\
\text { com }^{-}\end{array}$ & $\begin{array}{l}\text { Nota } \\
\text { mod }^{-}\end{array}$ \\
\hline Media_expte & $0,798^{* * *}$ & & & & & \\
\hline Nota_text & $0,252^{*}$ & $0,352^{* *}$ & & & & \\
\hline Nota_idioma & $0,312^{* *}$ & $0,337^{* *}$ & $0,273^{* *}$ & & & \\
\hline Nota_com & $0,575^{* * *}$ & $0,595^{* * *}$ & $0,361^{* * *}$ & $0,185^{*}$ & & \\
\hline Nota_mod & $0,574^{* * *}$ & $0,617^{* * *}$ & $0,322^{* *}$ & $0,152 \mathrm{~ns}$ & $0,368^{* * *}$ & \\
\hline Nota_fase_específica & $0,703^{* * *}$ & $0,617^{* * *}$ & $0,182 \mathrm{~ns}$ & $0,347^{* *}$ & $0,391^{* * *}$ & $0,525^{* * *}$ \\
\hline
\end{tabular}

${ }^{*} \mathrm{p}<0,05 ;{ }^{* *} \mathrm{p}<0,01 ;{ }^{* *} \mathrm{p}<0,001 ;$ ns: correlación no significativa.

TABLA 4: CORRELACIONES DE ORDEN CERO ENTRE EL RENDIMIENTO EN EL PRIMER CUATRIMESTRE DE PRIMERO DEL Grado en Biología y el Rendimiento previo (NOtAs de Bachillerato y PAU)

\begin{tabular}{|l|c|c|c|c|c|c|}
\hline Grado en Biología & $\begin{array}{c}\text { Rendi_ } \\
\text { alumno }\end{array}$ & $\begin{array}{c}\text { Media_ } \\
\text { expte }\end{array}$ & $\begin{array}{c}\text { Nota_ } \\
\text { text }\end{array}$ & $\begin{array}{c}\text { Nota_ } \\
\text { idioma }\end{array}$ & $\begin{array}{c}\text { Nota_ } \\
\text { com }\end{array}$ & $\begin{array}{c}\text { Nota } \\
\text { mod }\end{array}$ \\
\hline Media_expte & $0,462^{* * *}$ & & & & & \\
\hline Nota_text & $0,200^{*}$ & $0,255^{* *}$ & & & & \\
\hline Nota_idioma & $0,101 \mathrm{~ns}$ & $0,401^{* * *}$ & $0,140 \mathrm{~ns}$ & & & \\
\hline Nota_com & $0,298^{* *}$ & $0,241^{* *}$ & $0,136 \mathrm{~ns}$ & $-0,064 \mathrm{~ns}$ & & \\
\hline Nota_mod & $0,408^{* * *}$ & $0,211^{*}$ & $0,004 \mathrm{~ns}$ & $0,220^{*}$ & $0,086 \mathrm{~ns}$ & \\
\hline Nota_fase_especifica & $0,451^{* * *}$ & $0,256^{* *}$ & $0,267^{* *}$ & $0,152 \mathrm{~ns}$ & $0,135 \mathrm{~ns}$ & $0,097 \mathrm{~ns}$ \\
\hline
\end{tabular}

${ }^{*} p<0,05 ;{ }^{* *} p<0,01 ;{ }^{* * *} p<0,001 ;$ ns: correlación no significativa.

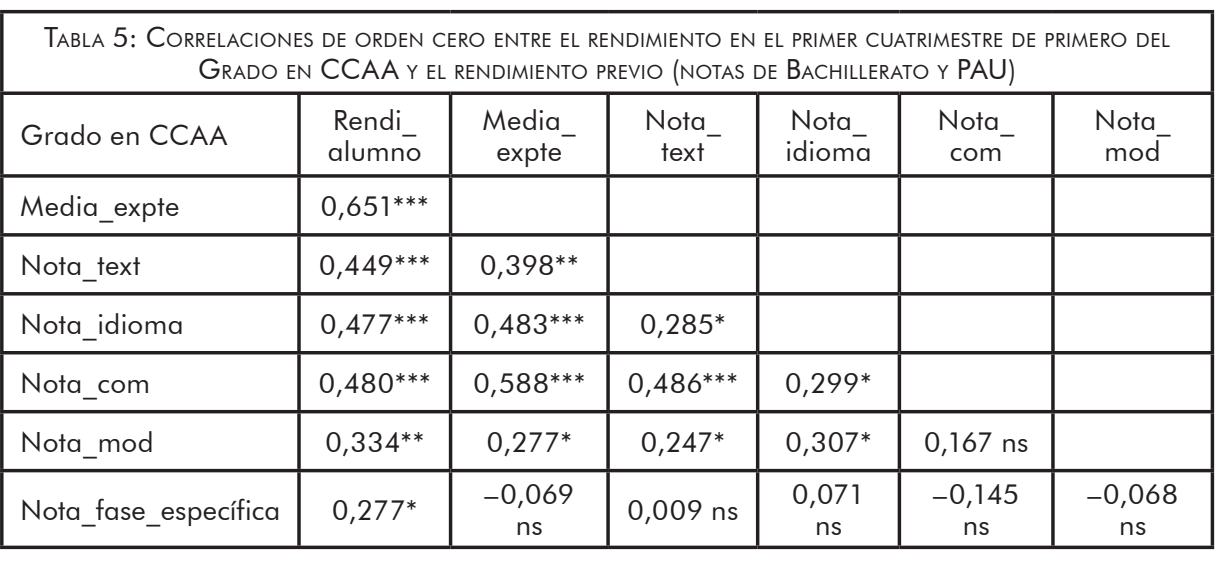

${ }^{*} \mathrm{p}<0,05 ;{ }^{* *} \mathrm{p}<0,01 ;{ }^{* * *} \mathrm{p}<0,001 ;$ ns: correlación no significativa. 
Efectivamente, tras realizar los procedimientos de selección de variables hacia adelante se confirma que todos incluyen en primer lugar a la variable nota media obtenida en el Bachillerato, por lo que este predictor es el que mejor explica la variabilidad del rendimiento futuro de un alumno.

Por otro lado, destaca que para todos los grados la nota de la fase específica también es elegida como variable a formar parte del modelo final. En concreto, esta variable se incluye en todos los modelos en el segundo paso excepto para el Grado en Matemáticas, donde lo hace en tercer lugar después de la nota de modalidad. Por lo tanto, se puede afirmar que la nota de la fase específica es también un predictor importante a la hora de estimar el rendimiento de un alumno en todos los grados.

En particular, para el Grado en Matemáticas, el modelo que mejor explica el rendimiento incluye en primer lugar la nota media obtenida en el Bachillerato, a continuación la nota de modalidad y, por último, la nota de la fase específica. Este modelo presenta un coeficiente de determinación ajustado $R_{\mathrm{aj}}^{2}=0,716$, es decir, que la variabilidad del rendimiento queda explicada en un $71,6 \%$ por dichas variables.

En la Tabla 6 se muestran los coeficientes para el modelo. Llevando a cabo una comparativa de los valores tipificados, $\beta$, se confirma que la nota media del expediente en el Bachillerato es la variable predictora dominante $\mid(\beta=0,504, p<0,001)$. Concretamente, la nota media en el Bachillerato explica ella sola el 63,2\% del rendimiento de los estudiantes del Grado en Matemáticas.

\begin{tabular}{|c|c|c|c|}
\hline \multicolumn{4}{|c|}{$\begin{array}{l}\text { Tabla 6: Coeficientes para el modelo predictivo del Rendimiento futuro de un } \\
\text { alumno en el Grado en MatemáticAs. }\end{array}$} \\
\hline Grado en Matemáticas & $b$ & Error típ. & $\beta$ \\
\hline Constante & $-29,479$ & 5,626 & \\
\hline Media_expte & 5,007 & 0,876 & $0,504^{* * *}$ \\
\hline Nota_mod & 1,562 & 0,395 & $0,287^{* * *}$ \\
\hline Nota_fase_específica & 2,036 & 0,832 & $0,204^{*}$ \\
\hline
\end{tabular}

${ }^{*} p<0,05 ;{ }^{* *} p<0,01 ;{ }^{* * *} p<0,001$.

Para el Grado en Química, el modelo final que se obtiene después de aplicar el método de selección de variables incluye a las mismas tres variables independientes que resultaron elegidas para el Grado en Matemáticas. La única diferencia es que en este caso entra en segundo lugar la nota de la fase específica, mientras que la nota de modalidad lo hace en tercer lugar. A partir del coeficiente de determinación ajustado podemos afirmar que la variabilidad del rendimiento queda explicada en un $64,3 \%$ por dichas variables. 
Si observamos los coeficientes $\beta$ que se obtienen para este modelo (Tabla 7) se concluye nuevamente que la nota media del expediente en el Bachillerato es la variable predictora más importante $\mid(\beta=0,525, p<0,001)$. En este caso, la variabilidad del rendimiento de los estudiantes del Grado en Química queda explicada en un $50,9 \%$ por la nota media en el Bachillerato.

\begin{tabular}{|l|c|c|c|}
\hline \multicolumn{4}{|c|}{$\begin{array}{c}\text { Tabla 7: Coeficientes para el mOdelo PRedictivo del Rendimiento } \\
\text { futuro de Un Alumno en el Grado en Química }\end{array}$} \\
\hline Grado en Química & $\mathrm{b}$ & Error típ. & $\beta$ \\
\hline Constante & $-29,340$ & 4,816 & \\
\hline Media_expte & 4,997 & 0,701 & $0,525^{* * *}$ \\
\hline Nota_fase_específica & 2,387 & 0,502 & $0,328^{* * *}$ \\
\hline Nota_mod & 1,215 & 0,344 & $0,247^{* *}$ \\
\hline
\end{tabular}

${ }^{*} p<0,05 ;{ }^{* *} p<0,01 ;{ }^{* * *} p<0,001$.

Con respecto al Grado en Física, el modelo obtenido sólo incluye a las variables nota media del expediente en el Bachillerato y nota de la fase específica. Además, el coeficiente de determinación ajustado es $R_{\mathrm{aj}}^{2}=0,702$ es decir, a partir de ambas variables se puede explicar el 70,2\% de la variabilidad del rendimiento.

$\mathrm{Al}$ igual que en los modelos anteriores, la Tabla 8 muestra que el mayor coeficiente $\beta$ es el de la nota media del expediente en el Bachillerato $(\beta=0,588, p<0,001)$, lo que corrobora que esta es la variable que más contribuye a explicar el rendimiento de los alumnos del Grado en Física, concretamente en un 63,3\%.

\begin{tabular}{|l|c|c|c|}
\hline \multicolumn{4}{|c|}{ Tabla 8: Coeficientes para el modelo predictivo del Rendimiento futuro de Un } \\
\hline Glumno en el Grado en Física
\end{tabular}

${ }^{*} \mathrm{p}<0,05 ;{ }^{* *} \mathrm{p}<0,01 ;{ }^{* * *} \mathrm{p}<0,001$.

El modelo que se obtiene para explicar el rendimiento en el Grado en Biología después de aplicar el método de selección de variables es el que incluye a más variables independientes: nota media obtenida en el Bachillerato, nota de la fase específica, nota de modalidad y nota en lengua extranjera. Sin embargo, 
es el modelo que presenta menor coeficiente de determinación ajustado $\left(R_{\mathrm{aj}}^{2}=0,428\right)$, aunque éste sigue siendo bastante alto y muestra que a partir de las variables anteriores se puede explicar el rendimiento futuro en un $42,8 \%$.

En esta ocasión, si comparamos los coeficientes $\square$ del modelo que se presentan en la Tabla 9, observamos que el coeficiente de la nota media del expediente en el Bachillerato sigue siendo ligeramente mayor que los demás $(\beta=0,372, p<0,001)$, consiguiendo esta variable explicar el 20,6\% del rendimiento. La inclusión de la nota de la fase específica incrementa el coeficiente de determinación ajustado hasta un $32 \%$.

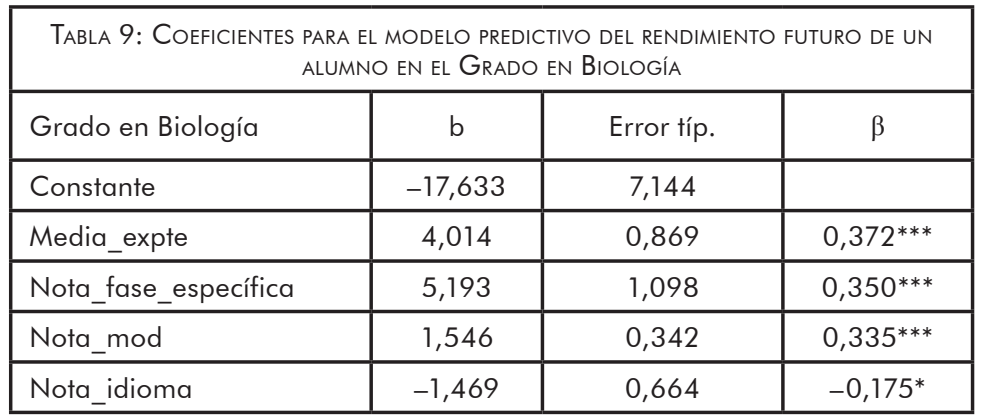

${ }^{*} p<0,05 ;{ }^{* *} p<0,01 ;{ }^{* * *} p<0,001$.

Por último, para el Grado en Ciencias Ambientales, tal y como ocurre para la mayoría de los modelos analizados, la nota media del expediente en el Bachillerato y la nota de la fase específica resultan ser las variables que más aportan a la hora de explicar el rendimiento. En este caso, el modelo final también incluye la variable nota en el comentario de texto, y a partir de las tres variables se logra explicar al rendimiento en un $54,2 \%$.

Una vez más, la comparación de los coeficientes tipificados $\beta$ del modelo en la Tabla 10 pone de manifiesto que la nota media del expediente en el Bachillerato vuelve a ser la variable predictora dominante $(\beta=0,589, p<0,001)$,explicando el $41,4 \%$ de la variabilidad del rendimiento.

\begin{tabular}{|c|c|c|c|}
\hline \multicolumn{4}{|c|}{$\begin{array}{c}\text { Tabla 10: Coeficientes para el modelo predictivo del Rendimiento futuro } \\
\text { DE un alumno en el Grado en CCAA }\end{array}$} \\
\hline Grado en CCAA & $b$ & Error típ. & $\beta$ \\
\hline Constante & $-30,626$ & 5,790 & \\
\hline Media_expte & 4,882 & 0,821 & $0,589^{* * *}$ \\
\hline Nota_fase_específica & 1,434 & 0,412 & $0,316^{* *}$ \\
\hline Nota_text & 1,064 & 0,495 & $0,212^{*}$ \\
\hline
\end{tabular}

${ }^{*} p<0,05 ;{ }^{* *} p<0,01 ;{ }^{* * *} p<0,001$. 
Cabe destacar que en todos los modelos se han identificado varios casos anómalos a partir del estudio de los residuales tipificados y la distancia de Mahalanobis pero, según el análisis de las distancias de Cook, ningún caso resulta ser influyente. Por tanto, se han mantenido todas las observaciones, ya que su eliminación no aporta ninguna mejoría notable a la bondad de ajuste del modelo.

Operativización. De acuerdo con los coeficientes de las Tablas 1-5, las ecuaciones que estiman el rendimento son:

- Para el Grado en Matemáticas:

Rendimiento $=-29,479+5,007 *$ Media_expte $+1,562 *$ Nota_mod + 2,036* Nota fase específica.

- Para el Grado en Química:

Rendimiento $=-29,340+4,997 *$ Media_expte $+2,387 *$ Nota_fase_específica $+1,215^{*}$ Nota_mod.

- Para el Grado en Física:

Rendimiento $=-39,150+6,484^{*}$ Media_expte $+4,230 *$ Nota_fase_específica.

- Para el Grado en Biología:

Rendimiento $=-17,633+4,014^{*}$ Media_expte $+5,193 *$ Nota_fase_específica $+1,546 *$ Nota_mod $-1,469 *$ Nota_idioma.

- Para el Grado en Ciencias Ambientales:

Rendimiento $=-30,626+4,882^{*}$ Media_expte $+1,434^{*}$ Nota_fase_específica $+1,064^{*}$ Nota_text.

Por lo tanto, para cada estudiante de nuevo ingreso habría que calcular su rendimiento estimado utilizando la ecuación correspondiente.

Aunque al comienzo se estableció que un alumno tiene un rendimiento bajo cuando el indicador toma un valor menor o igual a 10, proponemos que aquellos alumnos con un rendimiento estimado menor o igual a 15 reciban apoyo académico. De esta manera se evita que el procedimiento no detecte a determinados estudiantes con rendimiento bajo, aunque esto suponga que alumnos con rendimiento no tan bajo también sean marcados como alumnos necesitados de refuerzo académico.

\section{CONCLUSIONES}

Los resultados obtenidos indican que en todos los grados de la Facultad de Ciencias existe una relación preponderante del rendimiento previo como predictor del rendimiento futuro, medido a través de un nuevo indicador que resulta tener un alto poder discriminatorio. Así, el rendimiento futuro, definido como la suma de las notas de aquellas asignaturas aprobadas por un estudiante que se encuentra en el primer curso-primer cuatrimestre de cualquiera de los grados, está altamente correlacionado con la nota media del expediente en el Bachillerato (variable predictora dominante). Asimismo, 
la nota de la fase específica (PAU) se incluye en todos los modelos elaborados, lo que confirma que también es una variable importante a la hora de explicar el rendimiento futuro. Además, para los grados en Matemáticas, en Química y en Biología, la nota de la asignatura de modalidad (PAU) contribuye a mejorar la explicación del rendimiento futuro.

En general, se mantiene la consistencia con los resultados de Zeegers (2004) y de Rodríguez y Ruiz (2011). Estos autores también evaluaron el rendimiento en los estudios universitarios, pero considerando como índices de rendimiento el promedio de las calificaciones de un estudiante, por una parte, y la razón entre los créditos acumulados por el estudiante durante cierto período de tiempo y los créditos que teóricamente debió acumular en dicho período de acuerdo con el plan de estudios programado, por otra. En los dos casos quedó demostrada la dominancia del rendimiento previo como predictor de ambas formas de rendimiento futuro.

Consideramos que el modelo que proponemos es una herramienta de utilidad y gran valor predictivo que puede ser empleado por la universidad para detectar aquellos individuos susceptibles de ser orientados hacia la mejora de su aprovechamiento académico.

Haciendo uso del índice propuesto, la bondad de ajuste de los modelos de regresión lineal multivariante obtenidos (con valores de $R_{\text {aj }}^{2}$ entre 0,428 para el Grado en Biología y 0,716 para el Grado en Matemáticas) superan notablemente las bondades de ajuste de los diferentes modelos obtenidos por Rodríguez y Ruiz (2011) cuyo valor era de un $R^{2} \cong 0,390$, lo que confirma el alto poder discriminatorio de esta nueva medida del rendimiento.

Se llevaron a cabo análisis de regresión lineal multivariante adicionales incluyendo el género como variable independiente. Sin embargo, el género no resultó ser un predictor significativo del rendimiento académico en ninguno de los grados. Se condujeron además análisis de regresión lineal multivariante para hombres y mujeres por separado y los resultados fueron muy similares a los obtenidos para el conjunto de la muestra: en todos los modelos, el factor de predicción más significativo continuó siendo la nota media de Bachillerato. Por tanto, las ecuaciones propuestas pueden ser usadas para predecir el rendimiento académico de alumnos y alumnas indistintamente.

Cabría considerar otros factores utilizados en la investigación del rendimiento universitario, como podrían ser variables sociodemográficas, o introducir escalas como la de tendencias motivacionales personales de Hayamizu y Weiner (1991) o la escala de capacidad percibida de Trapnell (1994), ambas relacionadas con la percepción subjetiva que tiene el alumnado respecto a su formación. Aun así, consideramos que la introducción de este tipo de variables no aportaría ninguna mejora notable de la bondad de ajuste de nuestro modelo, por cuanto todas ellas subyacen al rendimiento escolar desde etapas tempranas y por tanto ya son implícitamente tenidas en cuenta al medir el éxito preuniversitario. 


\section{ANEXO}

A continuación se resumen el perfil y resultados académicos del alumnado de nuevo ingreso en la Facultad de Ciencias en los cursos 2015/16 y 2016/17 en función de la nota de admisión.
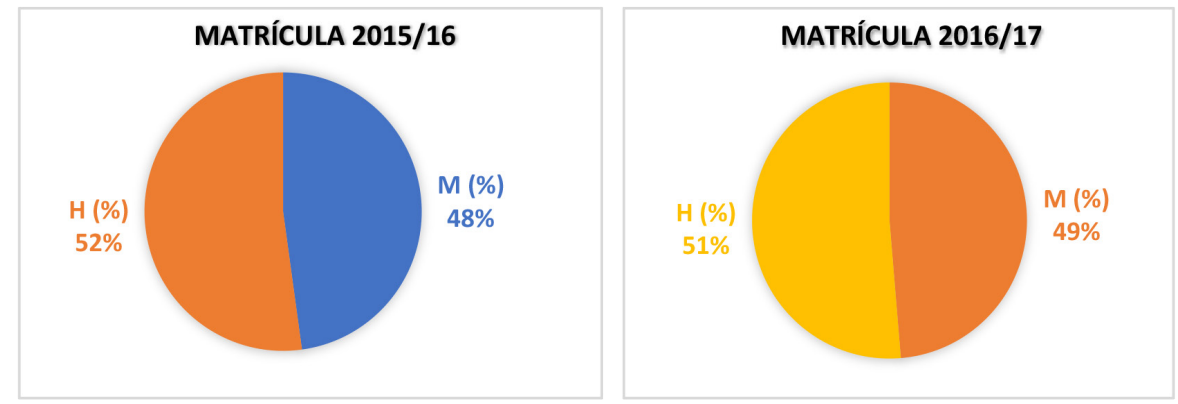

Gráfico 1. Datos de matrícula desagregados por género ( $H$ : hombres, $M$ : mujeres).

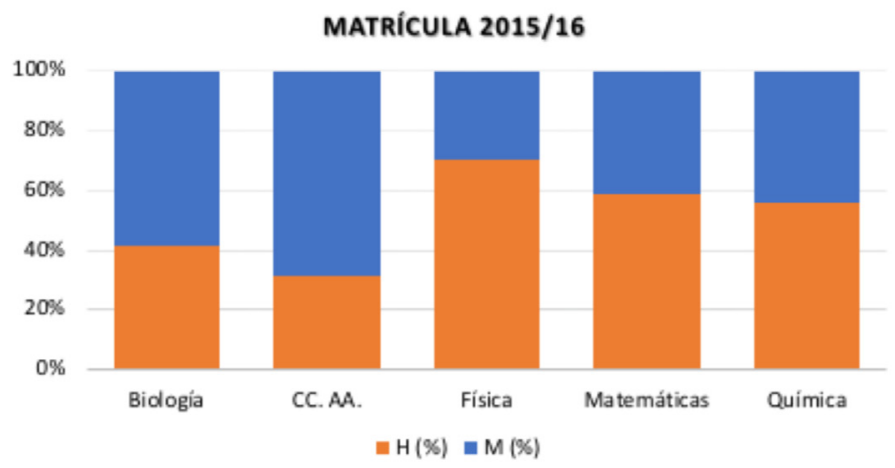

Gráfico 2. Datos de matrícula del curso 2015/16 desagregados por grado y género (H: hombres, M: mujeres).

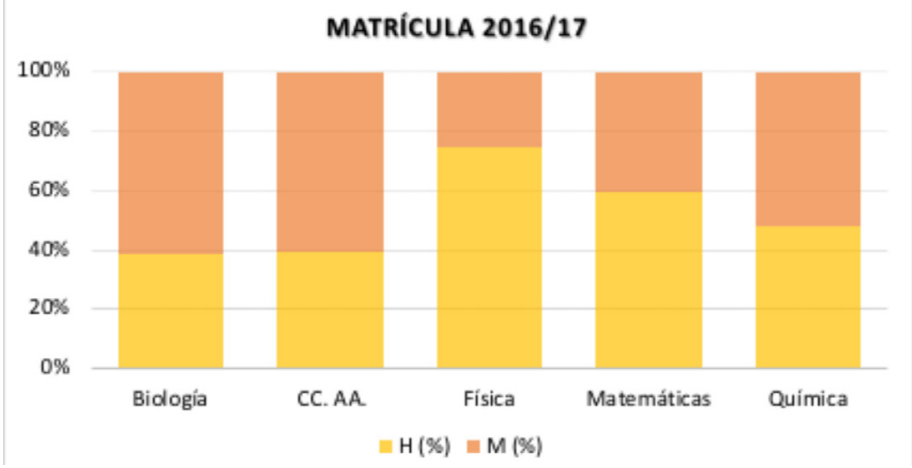

Gráfico 3. Datos de matrícula del curso 2016/17 desagregados por grado y género (H: hombres, $M$ : mujeres). 


\section{NOTA DE ADMISIÓN CURSO 2015/16}

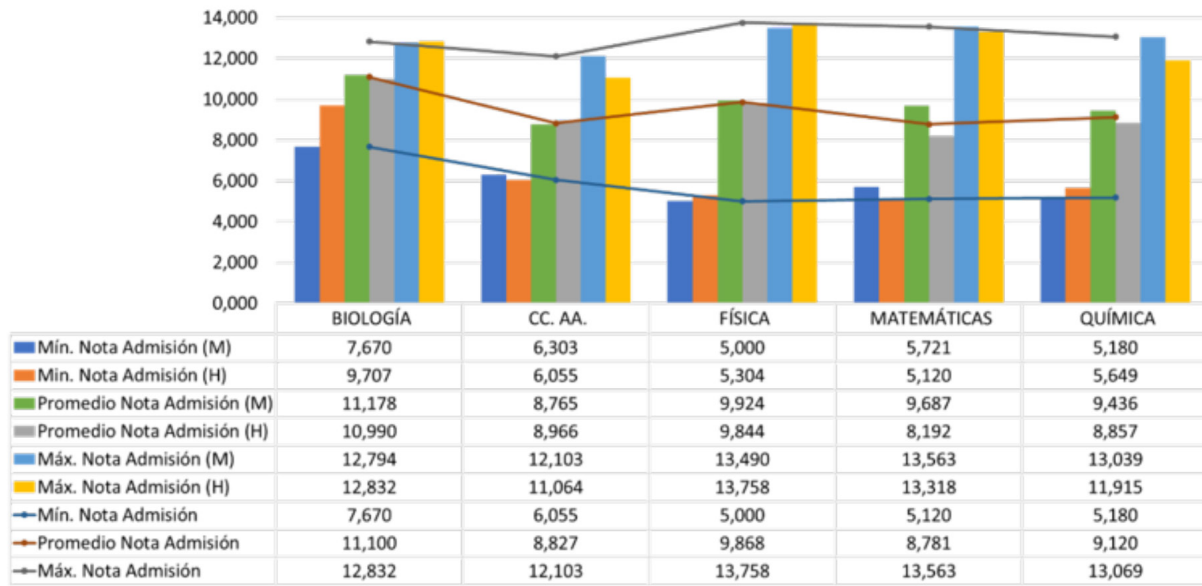

Gráfico 4. Notas de admisión del curso 2015/16 desagregadas por grado y género (H: hombres, M: mujeres).

\section{NOTA DE ADMISIÓN}

CURSO 2016/17

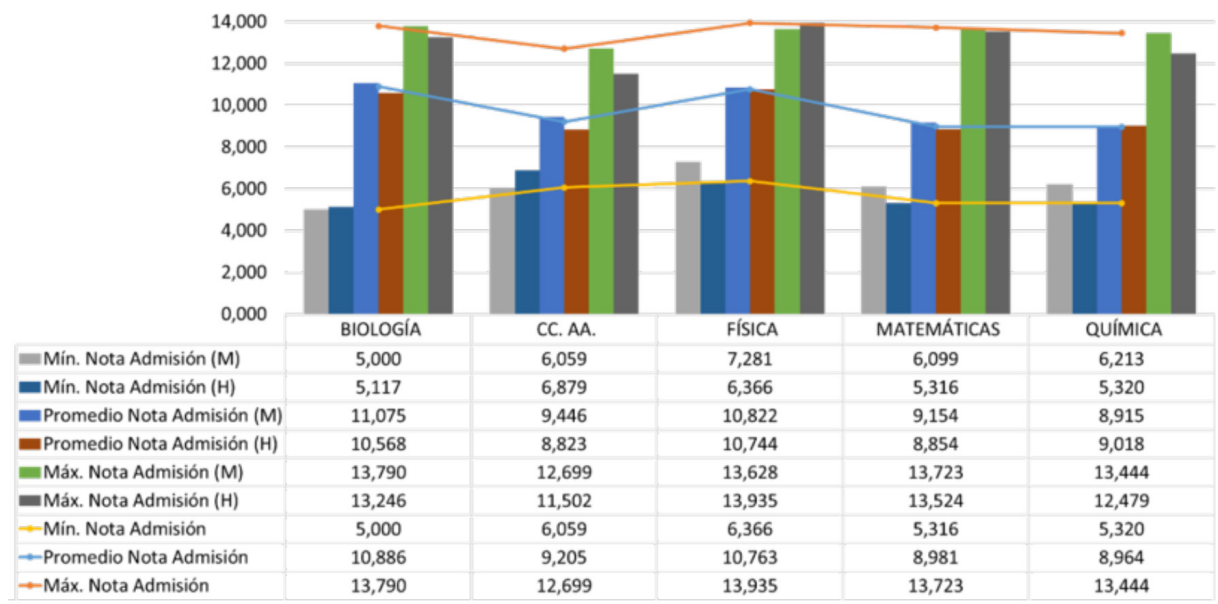

Gráfico 5. Notas de admisión del curso 2016/17 desagregadas por grado y género $(\mathrm{H}$ : hombres, M: mujeres). 
RESULTADOS DEL GRADO EN BIOLOGÍA

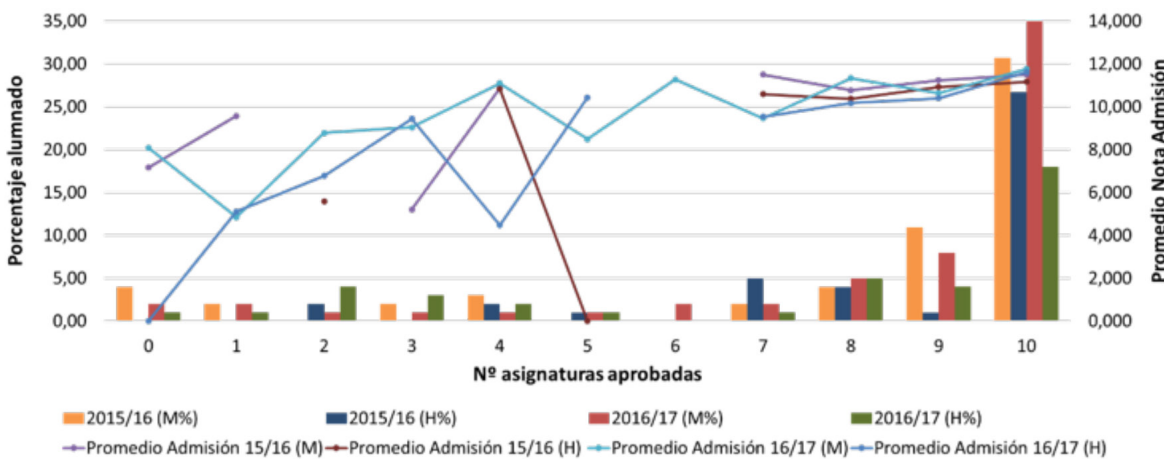

Gráfico 6. Resultados del Grado en Biología vs promedio de notas de admisión, desagregados por curso académico y género $(\mathrm{H}$ : hombres, $M$ : mujeres).

\section{RESULTADOS DEL GRADO EN CIENCIAS AMBIENTALES}

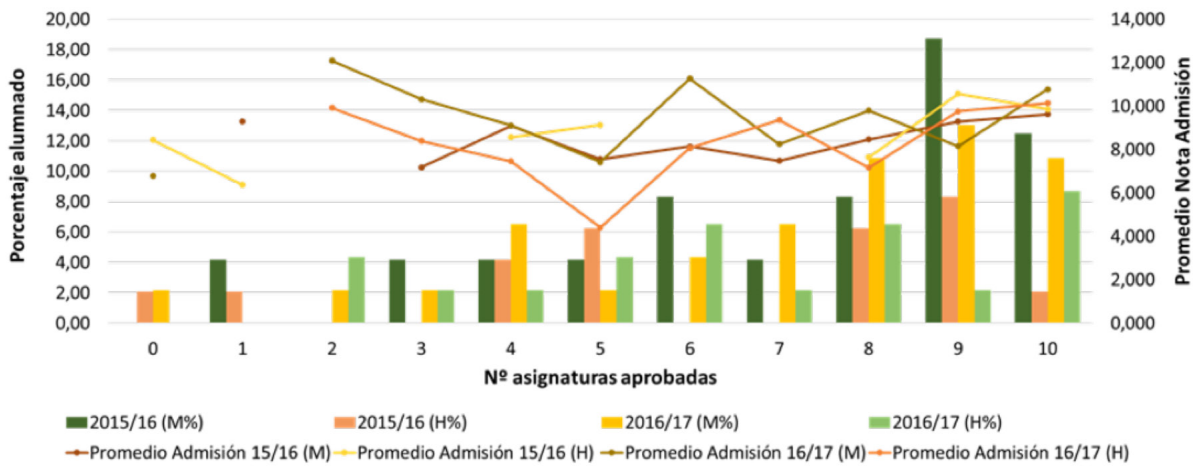

Gráfico 7. Resultados del Grado en Ciencias Ambientales vs promedio de notas de admisión, desagregados por curso académico y género ( $\mathrm{H}$ : hombres, $M$ : mujeres).

\section{RESULTADOS DEL GRADO EN FÍSICA}

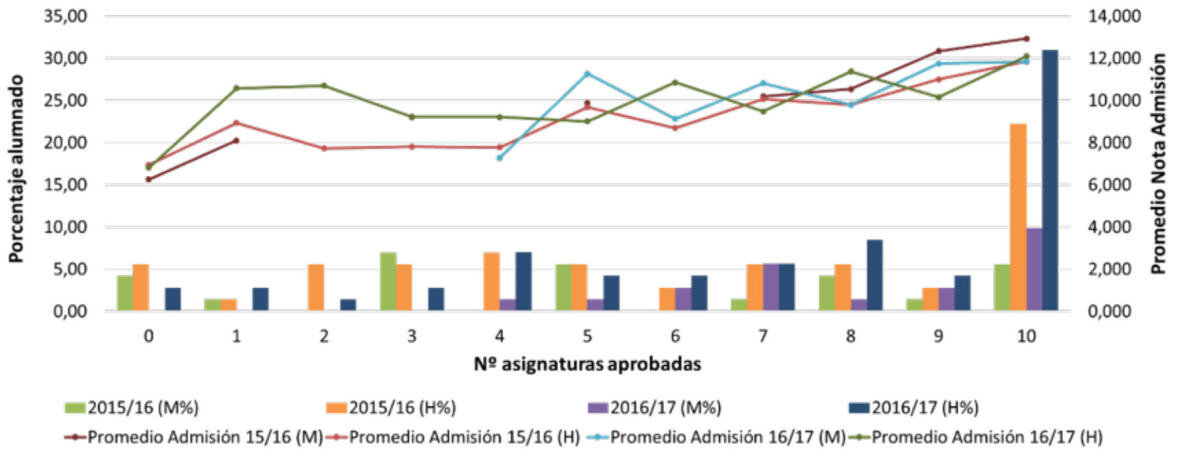

Gráfico 8. Resultados del Grado en Física vs promedio de notas de admisión, desagregados por curso académico y género ( $\mathrm{H}$ : hombres, $M$ : mujeres) 
RESULTADOS DEL GRADO EN MATEMÁTICAS

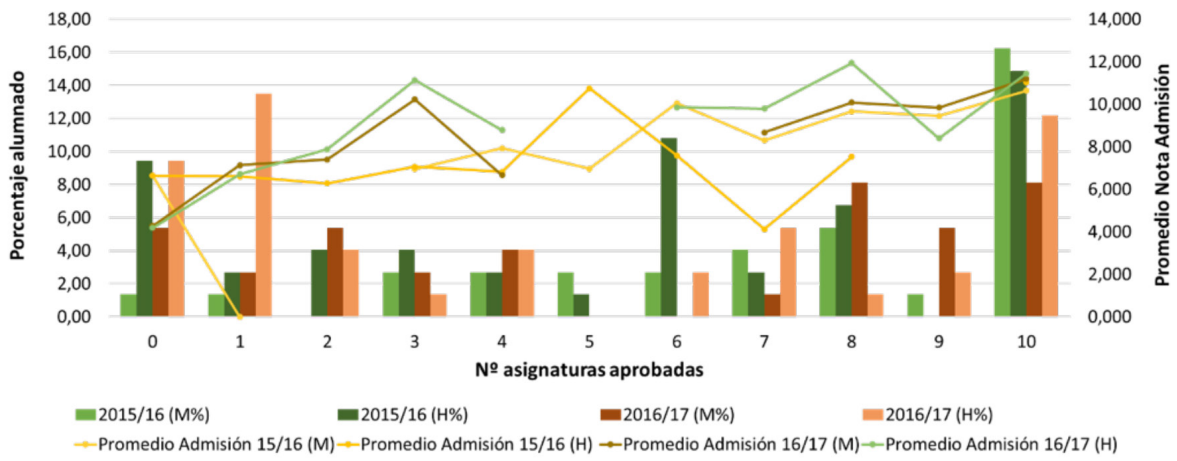

Gráfico 9. Resultados del Grado en Matemáticas vs promedio de notas de admisión, desagregados por curso académico y género ( $\mathrm{H}$ : hombres, $M$ : mujeres).

\section{RESULTADOS DEL GRADO EN QUÍMICA}

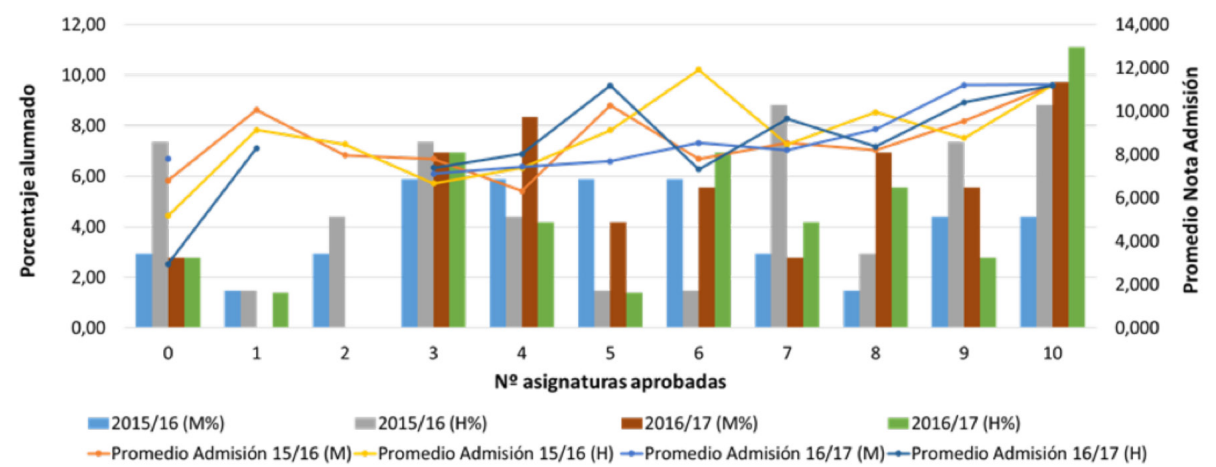

Gráfico 10. Resultados del Grado en Química vs promedio de notas de admisión, desagregados por curso académico y género ( $\mathrm{H}$ : hombres, $M$ : mujeres). 


\section{CONCLUSIONES (ANEXO).}

La matrícula de nuevo ingreso es paritaria en el conjunto de la Facultad de Ciencias. Por titulaciones, las mujeres ingresan en mayor proporción en Ciencias Ambientales y Biología, y los hombres en Física y Matemáticas.

La nota media de admisión es razonablemente buena, oscilando en torno a 9 en los grados en Ciencias Ambientales, Matemáticas y Química, 10 en el Grado en Física y 11 en el Grado en Biología.

El promedio de las notas de admisión de mujeres y hombres es muy similar en todos los casos. La máxima diferencia se encuentra en la cohorte 2015/16 de Matemáticas, donde la nota media de las mujeres supera a la de los hombres en 1,5 puntos.

Excepto en el Grado en Ciencias Ambientales se aprecia, como cabía esperar, una tendencia a la correlación positiva entre la nota media de admisión y el número de asignaturas aprobadas.

En ambos cursos académicos, aproximadamente el 55\% del alumnado de nuevo ingreso en Biología supera todo primero, mientras que un $60 \%$ del de Ciencias Ambientales aprueba entre 7 y 10 asignaturas. En el extremo opuesto, con un $35 \%$ y un $45 \%$ que suspende 7 o más asignaturas y un $10 \%$ y un $15 \%$ que ni siquiera aprueba una se sitúan las cohortes 2015/16 y 2016/17 de Química y Matemáticas, respectivamente. La proporción de quienes aprueban las 10 asignaturas del primer curso de Física oscila entre el $28 \%$ y el $40 \%$.

El conjunto de estudiantes que supera todo primero está com134 puesto mayoritariamente por mujeres en los casos de Biología y Ciencias Ambientales, y por hombres en los de Física y Química. En torno al 10\% de los alumnos, frente a un máximo del $5 \%$ de las alumnas, no aprueba ninguna asignatura de primero de Matemáticas.

\section{NOTA FINAL}

Salvo indicación expresa en contra, en todas las menciones de este documento a personas, colectivos, cargos académicos, etc. se está haciendo uso del sustantivo masculino gramatical para designar a todos los individuos sin distinción de sexo, englobando, en consecuencia, la posibilidad de referirse tanto a mujeres como a hombres. 


\section{AGRADECIMIENTOS}

Esta investigación ha estado parcialmente financiada por el Vicerrectorado de Docencia de la ULL en el marco del proyecto de innovación educativa titulado Plan de detección y atención de deficiencias formativas en Biología, Física, Geología, Matemáticas y Química para el alumnado de nuevo ingreso de la Facultad de Ciencias $\left(1^{\circ}\right.$ y $2^{\circ}$ ediciones, cursos 2016/17 y 2017/18). Agradecemos al Gabinete de Análisis y Planificación (GAP) de la ULL su diligencia en facilitarnos los datos estadísticos necesarios para desarrollarla. Todos los análisis, tablas y gráficos contenidos en el presente trabajo son de elaboración propia a partir de tales datos.

\section{REFERENCIAS BIBLIOGRÁFICAS}

BIVIN, D. y Rooney, P. (1999). Forecasting credit hours. Research in Higher Education, 40, 613-632.

HaYAmizu, T. y Weiner, B. (1991). A test of Dweck's model of achievement goals as related to perceptions of ability. Journal of Experimental Education, 59, 226-234.

MATHIASEN, R. L. (1984). Producing college academic achievement: A research review. College Student Journal, 18, 380-386.

PIKE, G.R. (1991). The effect of background, coursework and involvement on students' grades and satisfaction. Research in Higher Education, 32, 15-30.

PIKE, G.R. y SAUPE, J. (2002). Does high school matter? Research in Higher Education, 43, 187-207.

Rodríguez Ayán, M. N. y Ruiz Díaz, M. Á. (2011). Indicadores de rendimiento de estudiantes universitarios: calificaciones versus créditos acumulados. Revista de Educación, 355, 467-492.

TrapnelL, P. (1994). Openness versus intellect: A lexical left turn. European Journal of Personality, 8, 273-290.

Trujillo, R., Marrero, I., Dorta, R. y Torres, N. (2018). Relevancia e impacto del perfil formativo de los estudiantes de nuevo ingreso en los grados en Ciencias sobre su progreso y continuidad: Un modelo predictivo. En A. Vega y D. Stendardi (coords.): De la innovación imaginada a los procesos de cambio (Actas de las VIII Jornadas de Innovación Educativa, 2017), SPULL, pp. 279-298. 
